\title{
AUSTRALIA TELESCOPE OBSERVATIONS OF THE SUPERNOVA REMNANT N49 IN THE LMC
}

\author{
NORBERT JUNKES \\ Australia Telescope National Facility $\dagger$ \\ Epping, NSW, 2121 \\ Australia \\ Max-Planck-Institute für Radioastronomie \\ Auf dem Hügel 69 \\ 5300 Bonnl \\ F.R. Germany
}

$\dagger$ The Australia Telescope National Facility is operated in association with the Division of Radiophysics by CSIRO

\begin{abstract}
We present the first high-resolution radio image of the supernova remnant N49 near the northern edge of the LMC. These observations with the Australia Telescope at $6 \mathrm{~cm}$ wavelength (4 arcsec resolution) show a distinct radio shell. The shell structure of N49 is similar in radio and X-ray emission, with the maximum of the emission found in the southeastern part of the SNR.
\end{abstract}

These Australia Telescope (AT) observations of N49 (Henize, 1956) are the first results of a project to investigate the interaction of SNRs in the LMC with the interstellar medium (ISM) and the impact of this interaction on the formation of new stars.

About 25 of the compact unresolved radio sources in large-scale radio surveys of the LMC could be confirmed as supernova remnants (e.g. Mathewson et al. 1983). Whilst the study of SNRs in the Galaxy is seriously limited by gas, dust and galactic background emission which obscure our view of these objects, they can be observed, relatively unobscured, in the LMC. This is because this galaxy, the closest neighbour to our own, appears relatively face-on to us.

We selected candidates for SNR interaction with the ISM from their association with HII regions and OB associations in the LMC. N49 is an SNR at the edge of a giant shell (Shapley III) in the northern part of the LMC. This shell shows up in HI and optical filaments and contains a number of SNRs and HII regions.

Our radio image of $\mathrm{N} 49$ (fig. 1), resulting from observations in a $3 \mathrm{~km}$ configuration of the AT with a shortest baseline of $70 \mathrm{~m}$, contains a total flux density of $0.6 \mathrm{Jy}$, almost identical to the result from single-dish observations at the same wavelength (Milne et al. 1980).

The angular diameter of the radio image, about $1.4^{\prime}(22 \mathrm{pc}$ for an assumed distance of $55 \mathrm{kpc}$ for the LMC), is similar to the diameter of the X-ray source (Helfand \& Long 1979), whereas most of the optical radiation emerges from a smaller region of $0.8^{\prime}$ diameter which resembles an incomplete shell open to the west (Shull et al. 1985).

The comparison of all three wavelength regimes for N49 reveals the following picture. The optical radiation is strongly concentrated to the south-eastern part of the SNR. Both radio and X-ray images show a shell structure with maximum emission in the south-eastern part of the remnant and significantly weaker emission in the west. However, the positions of the emission maxima in radio and X-ray seem to be anticorrelated and indicate different locations for synchrotron emission and hot shocked gas in N49. A similar result has been found for N132D, another SNR in the LMC (Dickel and Milne, this meeting).

We have observed N49 in two additional frequencies of the $6 \mathrm{~cm}$ band and at $3 \mathrm{~cm}$ wavelength, and these data will be used to derive a detailed spectral index distribution across the source. High-resolution spectroscopy with the Australia Telescope ( $\mathrm{HI}, \mathrm{OH}, \mathrm{H}_{2} \mathrm{CO}$ ) will contribute to our knowledge of the atomic and molecular components of the interstellar medium around $\mathrm{N} 49$ and their interaction with the supernova remnant. 


\section{REFERENCES}

Helfand, D.J. \& Long, K.S.: (1979), Nature 282, 589

Henize, K.G.: (1956), Astrophys. J. Suppl. 2, 315

Mathewson, D.S., Ford, V.L., Dopita, M.A., Tuohy, I.R., Long, K.S. \& Helfand, D.J.: (1983), Astrophys. J. Suppl. 51, 345

Milne, D.K., Caswell, J.L. \& Haynes, R.F.: (1980), Mon. Not. R. astron. Soc. 191, 469

Shull, P., Dyson, J.E., Kahn, F.D. \& West, K.A.: (1985), Mon. Not. R. astron. Soc. 212 , 799

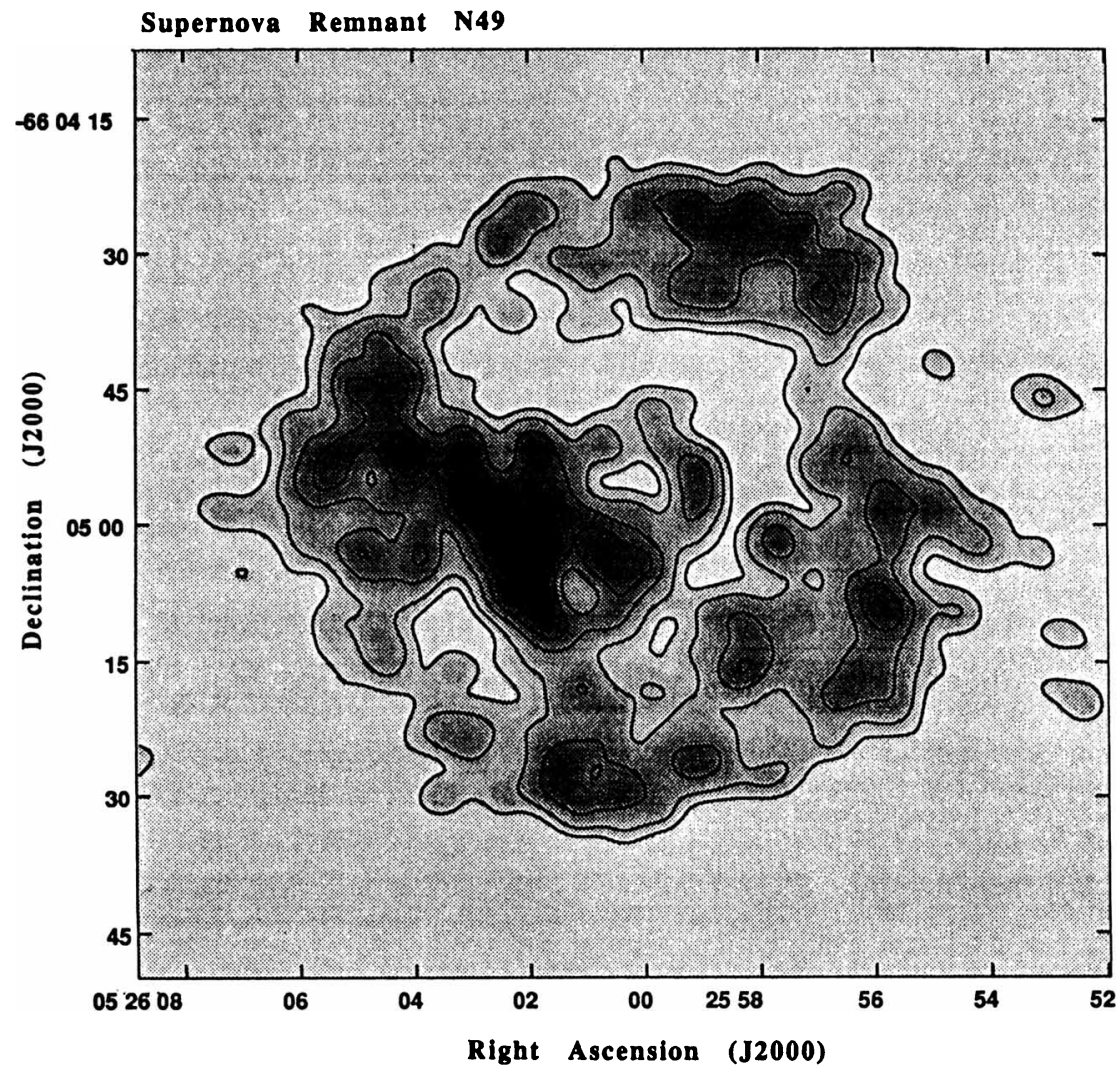

Fig. 1: First high-resolution radio image of the SNR N49 in the LMC. Observations at $6 \mathrm{~cm}$ wavelength in a $3 \mathrm{~km}$ configuration of the Australia Telescope (AT) provide a half-power beam width of $4.2 \times 3.8$ arcsecs. The SNR shows a shel: stricture with 1.4' angular diameter (22 pc at a distance of $55 \mathrm{kpc}$ for the LMC). The peak intensity is $14 \mathrm{mJy} / \mathrm{beam}$ and the total integrated flux about $0.6 \mathrm{Jy}$. The contour levels are 1,2,4,6, ..,16 mJy/beam and coordinates are given in the J2000 system. 